Supporting Information For

\title{
Exploring the Effects of Reaction Conditions on Morphology and Stability of Sonochemically Generated Ti-Al-B Fuel Powders
}

\author{
Matthew T. Finn ${ }^{1, *}$, Brian Chaloux ${ }^{2}$, Albert Epshteyn ${ }^{2, *}$ \\ ${ }^{1}$ ASEE Postdoctoral Fellow, Chemistry Division, Naval Research Lab, Washington, DC 20854 \\ ${ }^{2}$ Chemistry Division, Naval Research Lab, Washington, DC 20854
}

Corresponding Authors:

Matthew Finn and Albert Epshteyn, Naval Research Lab, Chemistry Division, 4555 Overlook

Ave., SW, Washington, DC 20375. matthew.finn.ctr@nrl.navy.mil; albert.epshteyn@nrl.navy.mil

Table of Contents

Table S1. Effective diameters of RMNP samples with different preparation methods and thermal treatment temperatures.

Figure S1. Wide area SEM image of traditional and reverse addition RMNP samples treated to $100^{\circ} \mathrm{C}$.

Figure S2. XPS spectra for the traditional and reverse addition RMNPs annealed at $150{ }^{\circ} \mathrm{C}$.

Figure S3. XPS spectra for the traditional and reverse addition RMNPs annealed at $200{ }^{\circ} \mathrm{C}$. 
Table S1. Effective diameters of RMNP samples with different preparation methods and thermal treatment temperatures. Data reported are an average of 5 scans after 10 minute sonication in $\mathrm{EtOH}$.

\begin{tabular}{cccc}
\hline & & Effective Diameter $(\mathbf{n m})$ & \\
RMNP Type & $\mathbf{1 0 0} \mathbf{C}$ & $\mathbf{1 5 0} \mathbf{~}^{\circ} \mathbf{C}$ & $\mathbf{2 0 0}^{\circ} \mathbf{C}$ \\
\hline \hline Traditional & 1,343 & 2,594 & 2,263 \\
Reverse & 465 & 457 & 446 \\
\hline
\end{tabular}
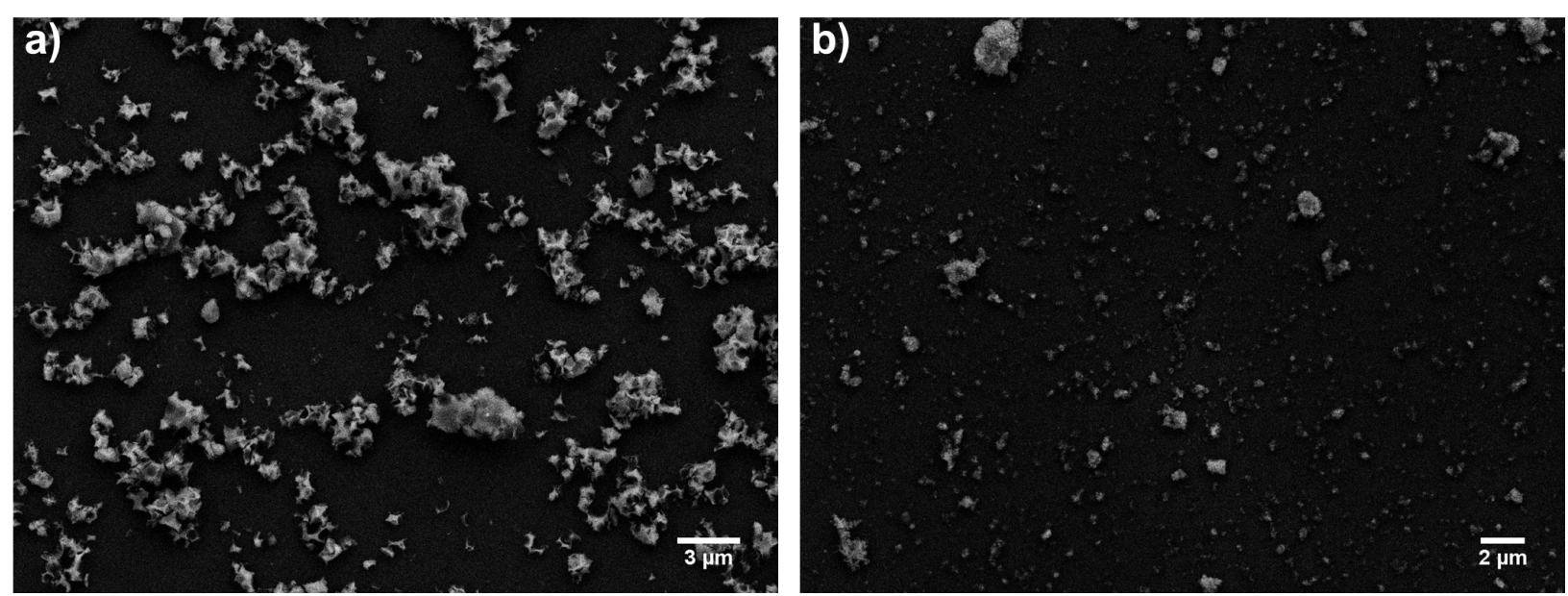

Figure S1. Wide area SEM image of traditional and reverse addition RMNP samples treated to $100^{\circ} \mathrm{C}$.
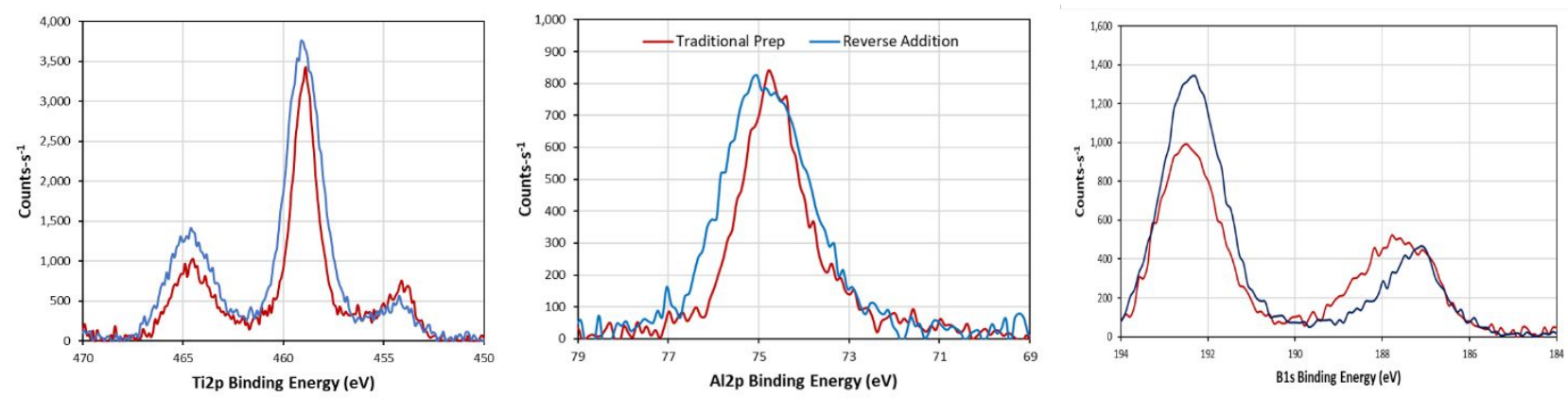

Figure S2. XPS spectra for the traditional and reverse addition RMNPs annealed at $150{ }^{\circ} \mathrm{C}$. 

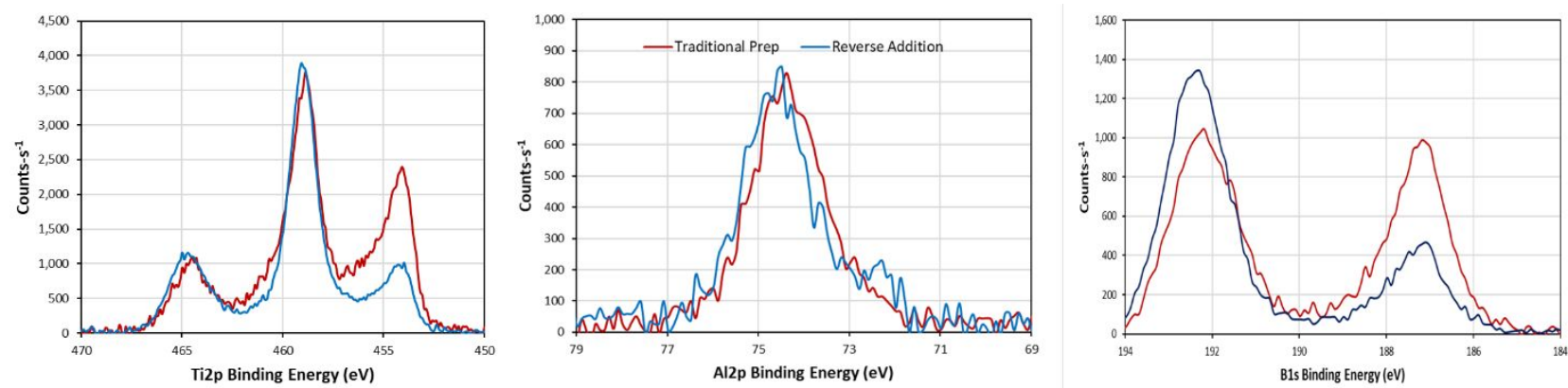

Figure S3. XPS spectra for the traditional and reverse addition RMNPs annealed at $200^{\circ} \mathrm{C}$. 